\title{
Uso da vitamina D para a diminuição do risco cardiovascular em pacientes com diabetes mellitus tipo II: Uma Revisão Sistemática
}

Use of vitamin D to decrease cardiovascular risk in patients with type II diabetes mellitus: A Systematic Review

Uso de vitamina D para disminuir el riesgo cardiovascular en pacientes con diabetes mellitus tipo II: Una revisión sistemática

Recebido: 06/06/2021 | Revisado: 14/06/2021 | Aceito: 16/06/2021 | Publicado: 30/06/2021

Rickardo Baia Diniz ORCID: https://orcid.org/0000-0002-6960-617X Centro Universitário de Patos, Brasil E-mail: rickardodiniz@ fiponline.edu.br

Tiago Bruno Carneiro de Farias ORCID: https://orcid.org/0000-0002-0473-1244 Centro Universitário de Patos, Brasil E-mail: tiagofarias@fiponline.edu.br

Milena Nunes Alves de Sousa

ORCID: https://orcid.org/0000-0002-9431-6326 Centro Universitário de Patos, Brasil E-mail: milenanunes@fiponline.edu.br

\begin{abstract}
Resumo
Introdução: A diabetes mellitus é uma doença cada vez mais prevalente no mundo moderno, sendo altamente relacionada a fatores genéticos e comportamentais e que acarreta em inúmeros problemas de saúde pública. Objetivo: analisar a utilização da vitamina D com o intuito de diminuir o risco cardiovascular em pacientes portadores de diabetes mellitus tipo 2. Métodos: Foi feita uma revisão sistemática da literatura. Para elaboração do estudo, realizou-se busca de dados nacionais e internacionais e sucedeu-se a eleição de critérios de elegibilidade considerando idioma do artigo (inglês e português), textos completos e publicados nos últimos 10 anos. Em sequência, a partir da leitura dos títulos e posterior análise dos resumos, a amostra final constituiu-se de 13 publicações. Resultados: Foi detectado um baixo número de publicações, contudo, constatou-se que a vitamina $\mathrm{D}$ e seus análogos têm uma eficácia direta sobre os marcadores utilizados para predizer o risco cardiovascular dos pacientes mesmo possuindo ainda dúvidas sobre seus mecanismos de ação. Conclusões: A intervenção com vitamina D apresenta bons resultados no tocante à diminuição dos marcadores de risco cardiovascular em pacientes diabéticos. Contudo, devem ser realizadas mais pesquisas para melhor esclarecer a correlação dos níveis de vitamina D e a diminuição de eventos cardiovasculares, além de melhor seus mecanismos fisiológicos, estratégias terapêuticas e seus efeitos nos mais diversos perfis populacionais para assim elaborar intervenções eficazes e conclusivas.
\end{abstract}

Palavras-chave: Vitamina D; Doença cardiovascular; Diabetes mellitus.

\begin{abstract}
Introduction: Diabetes mellitus is an increasingly prevalent disease in the modern world, being highly related to genetic and behavioral factors, causing numerous public health problems. Objective: analyze the use of vitamin D in order to reduce cardiovascular risk in patients with type 2 diabetes mellitus. Methods: A systematic review of the literature was performed. To prepare the study, national and international data were searched and the eligibility criteria were chosen considering the language of the article (English and Portuguese), complete texts and published in the last 10 years. In sequence, from the reading of the titles and subsequent analysis of the abstracts, the final sample consisted of 13 publications. Results: A low number of publications was detected, however, it was found that vitamin $\mathrm{D}$ and its analogs have a direct efficacy on the markers used to predict the cardiovascular risk of patients even though they still have doubts about their mechanisms of action. Conclusions: Intervention with vitamin D shows good results regarding the reduction of cardiovascular risk markers in diabetic patients. However, more research should be carried out to better clarify the correlation of vitamin D levels and the reduction of cardiovascular events, in addition to better their physiological mechanisms, therapeutic strategies and their effects on the most diverse population profiles in order to develop effective and conclusive interventions.
\end{abstract}

Keywords: Vitamin D; Cardiovascular disease; Diabetes mellitus. 


\begin{abstract}
Resumen
Introducción: La diabetes mellitus es una enfermedad cada vez más prevalente en el mundo moderno, estando altamente relacionada con factores genéticos y conductuales y causando numerosos problemas de salud pública. Objetivo: analizar el uso de vitamina D para reducir el riesgo cardiovascular en pacientes con diabetes mellitus tipo 2. Métodos: Se realizó una revisión sistemática de la literatura. Para la elaboración del estudio se realizó una búsqueda de datos nacionales e internacionales, seguida de la elección de los criterios de elegibilidad considerando el idioma del artículo (inglés y portugués), textos completos y publicados en los últimos 10 años. En secuencia, a partir de la lectura de los títulos y posterior análisis de los resúmenes, la muestra final estuvo conformada por 13 publicaciones. Resultados: Se detectó un número bajo de publicaciones, sin embargo, se encontró que la vitamina $\mathrm{D}$ y sus análogos tienen una eficacia directa sobre los marcadores utilizados para predecir el riesgo cardiovascular de los pacientes, aunque aún existen dudas sobre sus mecanismos de acción. Conclusiones: La intervención con vitamina D tiene buenos resultados en cuanto a reducción de marcadores de riesgo cardiovascular en pacientes diabéticos. Sin embargo, se deben realizar más investigaciones para esclarecer mejor la correlación entre los niveles de vitamina D y la disminución de eventos cardiovasculares, además de mejorar sus mecanismos fisiológicos, estrategias terapéuticas y sus efectos en los más diversos perfiles poblacionales, con el fin de diseñar efectivos y intervenciones concluyentes.
\end{abstract}

Palavras-chave: Vitamin D; Doença cardiovascular; Diabetes Mellitus.

\title{
1. Introdução
}

A Diabetes Mellitus Tipo II (DM2) é uma das doenças crônicas não transmissíveis mais prevalentes no cenário atual, encontrada nas mais diversas classes sociais, em ambos os sexos e em diferentes idades (Carrillo-Larco \& Bernabé-Ortiz, 2019). As Diretrizes da Sociedade Brasileira de Diabetes conceituam a DM2 como uma condição clínica multifatorial, decorrente de um conjunto de alterações metabólicas que acarretam na hiperglicemia persistente (glicemia de jejum maior ou igual a 126 $\mathrm{mg} / \mathrm{dL}$ ), devido à insulinodeficiência, insulinoresistência ou ambas (SBD, 2019-2020).

Dentre os impactos dessa condição estão a elevada prevalência, expressiva morbimortalidade decorrente de complicações agudas e crônicas e a alta taxa de hospitalizações, gerando significativos danos sociais e econômicos Aguiar, (Aguiar, Duarte \& Carvalho, 2019; Gondim, Oliveira \& Grossi, 2003; Lu et al., 2014; Odutayo et al., 2016). Dados da Federação Internacional de Diabetes (IDF) referem que no ano de 2013, o número de portadores de diabetes era de 387 milhões de pessoas, 46\% delas sem diagnóstico prévio. Em nível de América Central e América do Sul, a estimativa estava em torno de 24 milhões de pessoas, podendo chegar a 38,5 milhões em 2035 - um aumento em torno de 60\%. Para o Brasil, o número estimado de 11,9 milhões de casos pode alcançar 19,2 milhões em 2035 (Ruff et al., 2014).

A DM2 é frequentemente associada a outras patologias ou complicações, sendo apontada como fator capaz de ocasionar e agravar doenças cardiovasculares ateroscleróticas e, por consequência, provocar problemas cardíacos graves. Nesse sentido, também pode ser um fator preditor para o comprometimento miocárdico conhecido como cardiomiopatia diabética, tendo por consequência um risco duas a quatro vezes maior de insuficiência cardíaca (IC) em comparação com indivíduos não diabéticos (Sharma et al., 2018; Steele et al., 2020).

Concomitante a isso, foi apontado um risco duas vezes maior de infarto agudo do miocárdio, bem como de aterosclerose, em pacientes diabéticos em comparação a pacientes não diabéticos (Strain, \& Paldánius, 2018, Wacker \& Hollick, 2013).

Com o intuito de reduzir a morbimortalidade decorrente da DM2 diversas estratégias têm sido desenvolvidas visando desde o rastreamento precoce até o controle dos impactos causados por esta patologia (Wacker \& Hollick, 2013; Annweiler et al., 2014; Hirschler, et al., 2015).

Nesse contexto, a vitamina D tem sido apontada como uma alternativa terapêtica promissora. Trata-se de um importante pró-hormônio esteroide lipofílico, conhecido por seu papel fundamental na homeostase do cálcio e do fósforo e saúde musculoesquelético, mas que possui múltiplas funções fisiológicas, atuando na diferenciação e proliferação celular, secreção hormonal, no sistema imune e em diversas doenças crônicas não transmissíveis (Wacker \& Hollick, 2013; Mcdonnell et al., 2016; Muscogiuri et al., 2016; Zittermann et al., 2018). 
Vale ressaltar que, atualmente a hipovitaminose D tem sido apontada como um problema de saúde pública mundial, devido a sua implicação no desenvolvimento de vários distúrbios não esqueléticos, incluindo doenças endocrinometabólicas, doenças autoimunes e aumento do risco cardiovascular (Liefaard et al., 2015; Nakashima et al., 2016 \& Verdoia et al., 2020).

Com isso, o objetivo dessa revisão é analisar a utilização da vitamina D com o intuito de diminuir o risco cardiovascular em pacientes portadores de diabetes mellitus tipo 2 por meio da seguinte questão de pesquisa: "Quais os benefícios da suplementação de vitamina D na diminuição do risco cardiovascular em pacientes com diabetes mellitus tipo 2?”.

\section{Metodologia}

Foi conduzida uma revisão sistemática com análise qualitativa para determinar o impacto do uso da vitamina D em fatores cardiovasculares em pacientes com diabetes mellitus 2, por meio de resumo de evidências associadas a um método de intervenção específica. Assim, foi utilizada a estratégia PICO, na qual a população de interesse é composta por pessoas com diabetes mellitus 2 e a intervenção é o uso de vitamina D; o comparador é o tratamento usual sem a vitamina D e o objetivo ou desfecho é a diminuição dos eventos cardiovasculares.

Revisão sistemática da literatura é uma modalidade de pesquisa que segue protocolos específicos, buscando entender e fornecer logicidade a determinado tema, verificando o que funciona ou não dentro da literatura. Apresenta de forma explícita as bases de dados bibliográficos que foram consultadas, as estratégias de busca empregadas em cada base, o processo de seleção dos artigos científicos, os critérios de inclusão e exclusão dos artigos e o processo de análise de cada artigo. Além disso, explicita ainda as limitações de cada artigo analisado, bem como as limitações da própria revisão. De forma geral, a revisão de literatura sistemática possui alto nível de evidência e se constitui em um importante documento para tomada de decisão nos contextos públicos e privados (Galvão \& Ricarte, 2019).

Segundo Galvão e Ricarte (2019) as revisões sistemáticas da literatura devem ser construídas seguindo os seguintes passos:

1 - Delimitação da questão de pesquisa: essa etapa é essencial para que as outras sejam realizadas. A partir disso, formulouse a seguinte questão de pesquisa: "Quais os benefícios da suplementação de vitamina D na diminuição do risco cardiovascular em pacientes com diabetes mellitus tipo 2?".

2 - Seleção das bases de dados: definiu-se quais bases de dados seriam consultadas para a busca de artigos e outros materiais bibliográficos que possam ser incluídos ou excluídos da revisão de literatura que se pretende realizar. O conteúdo dessa pesquisa foi alcançado nas bases de dados PubMed, Science Direct e Ebsco Host.

3 - Elaboração da estratégia de busca: essa etapa tem o objetivo de encontrar os estudos mais relevantes publicados sobre determinado tema. Para isso, utilizaram-se os seguintes descritores em ciências da saúde (DECS): "Vitamin D"; "Cardiovascular diseases" e "Diabetes Mellitus", as quais foram relacionadas entre si com o uso do operador booleano "AND". Os filtros utilizados na pesquisa foram o tipo de publicação (clinical trial), idioma original do artigo (Inglês ou Português); texto completo disponível e trabalhos dos últimos 10 anos. Nesse sentido, para auxiliar na verificação dos critérios mínimos de qualidade das revisões de literatura, antes, durante e após publicação, adotou-se a recomendação PRISMA, como apresentado no Fluxograma 1 (PRISMA, 2018).

4 - Seleção das informações encontradas: realizou-se uma seleção e leitura integral dos artigos, buscando os seguintes dados: grupo controle, intervenção com vitamina D e comparativo entre os quadros clínicos desses pacientes. Contudo, foram excluídos artigos que estavam fora da temática estudada, com metodologia sem boa definição, uso de outras intervenções e publicações repetidas e duplicadas. Em sequência, vários ensaios clínicos randomizados foram avaliados, com grupos portadores de Diabetes Mellitus 2 e que receberam intervenção a Vitamina D. O intuito foi o de analisar a eficácia dessa terapêutica na clínica dos pacientes em comparação com uso de placebo. As principais alterações identificadas foram agrupadas no Quadro 2. 
5 - Sistematização das informações encontradas: Assim, por meio da leitura integral da amostra final, foi possível extrações de dados sendo incluído as seguintes informações: autor, ano, título, país, periódico, cenário de estudo, base de dados e nível de evidência. Tais informações estão evidenciadas no quadro 1. Juntamente a isso, também foram verificadas as informações sobre manifestações clínicas, idade dos pacientes, tamanho das amostras, de acordo com a recomendação PRISMA. Em conclusão, foi feita a síntese dos achados encontrados nos estudos selecionados.

Além disso, é essencial classificar o nível de evidência dos ensaios clínicos randomizados que foram selecionados para compor a amostra final. Para tanto, utilizou-se as Diretrizes Metodológicas do Sistema GRADE (Brasil, 2014). De acordo com essa ferramenta, esse tipo de estudo deve iniciar a análise com qualidade de evidência alta e, a partir da identificação ou não dos critérios que diminuem o nível de evidência (limitação metodológica, inconsistência nos resultados, evidência indireta e imprecisão dos resultados) devem ter sua qualidade reduzida.

No estudo em questão, os treze ensaios clínicos randomizados foram analisados quanto ao seu nível de evidência, apresentado no Quadro 3, e foram classificados com alto nível de evidência, tendo em vista que os fatores que reduzem sua qualidade não estavam presentes, qualificando os estudos como claros, objetivos e concisos com seus achados.

Fluxograma 1 - Esquema de seleção dos estudos para a revisão sistemática.

7004 estudos potencialmente elegíveis após inserção dos DeCS nas Bases de Dados

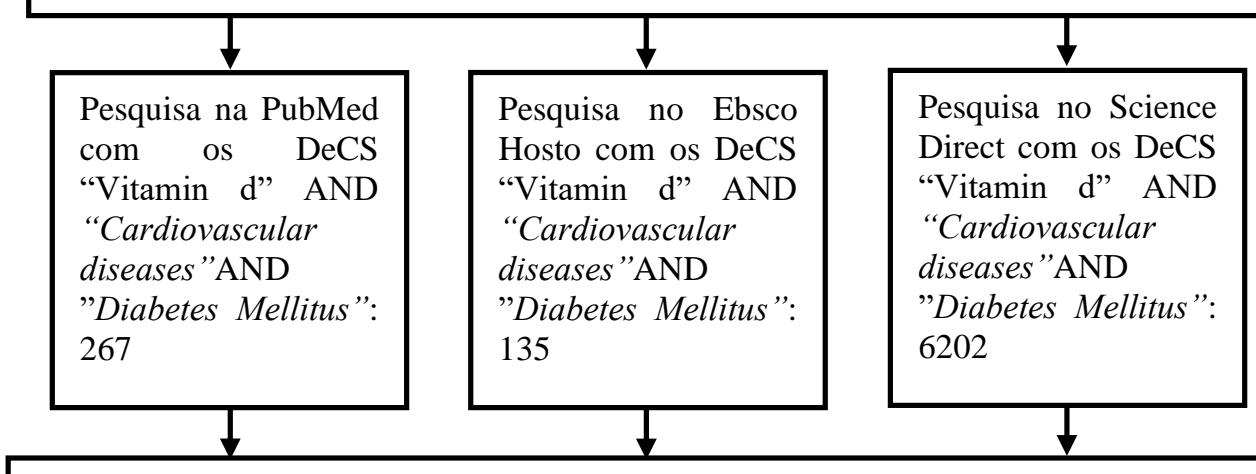

Aplicação dos filtros: Idioma inglês ou português; texto completo disponível; Ensaios clínicos randomizados; Estudos em humanos.

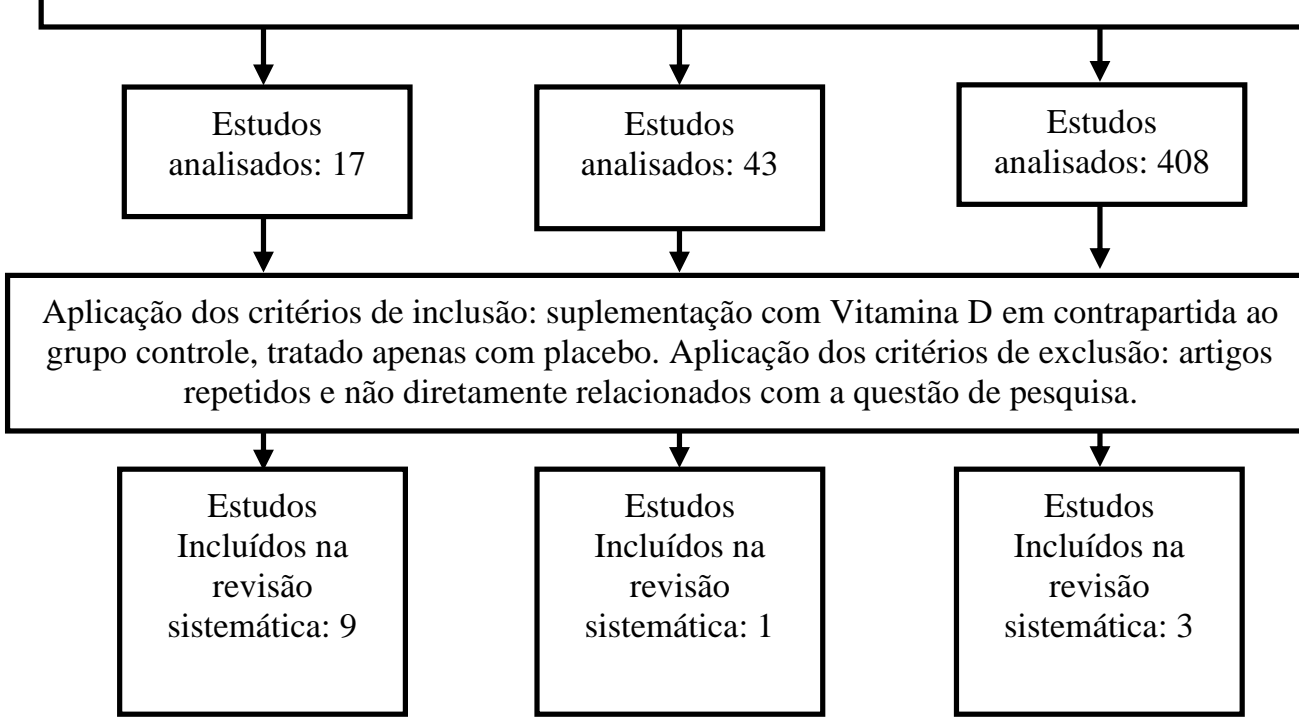

Fonte: Autores. 


\section{Resultados}

Assim, a princípio foi feita a sistematização dos estudos selecionados, classificando-os por autor/ano, título, país periódico, cenário de estudo e base de dados, representados pelo Quadro 1.

Quadro 1 - Descrição dos estudos selecionados para a revisão sistemática.

\begin{tabular}{|c|c|c|c|c|c|}
\hline Autor/Ano & Título & País & Periódico & $\begin{array}{l}\text { Cenário do } \\
\text { Estudo }\end{array}$ & Base de dados \\
\hline $\begin{array}{l}\text { Angelloti et al } \\
\text { (2018) }\end{array}$ & $\begin{array}{l}\text { Effect of vitamin D supplementation on } \\
\text { cardiovascular risk in type } 2 \text { diabetes. }\end{array}$ & EUA & $\begin{array}{l}\text { Clinical } \\
\text { Nutrition }\end{array}$ & $\begin{array}{l}\text { Ensaio Clinico } \\
\text { Randomizado }\end{array}$ & PubMed \\
\hline $\begin{array}{l}\text { Zaher et al } \\
\text { (2018) }\end{array}$ & $\begin{array}{l}\text { A randomized intervention study to } \\
\text { evaluate the effect of calcitriol therapy } \\
\text { on the renin-angiotensin system in } \\
\text { diabetes }\end{array}$ & EUA & Sabe Journals & $\begin{array}{l}\text { Ensaio Clinico } \\
\text { Randomizado }\end{array}$ & PubMed \\
\hline $\begin{array}{l}\text { E. Riek et al } \\
\text { (2017) }\end{array}$ & $\begin{array}{l}\text { Vitamin D3 supplementation decreases } \\
\text { a unique circulating monocyte } \\
\text { cholesterol pool in patients with type } 2 \\
\text { diabetes }\end{array}$ & EUA & $\begin{array}{l}\text { Journal of } \\
\text { Steroid } \\
\text { Biochemistry } \\
\text { and Molecular } \\
\text { Biology }\end{array}$ & $\begin{array}{l}\text { Ensaio Clinico } \\
\text { Randomizado }\end{array}$ & PubMed \\
\hline $\begin{array}{l}\text { Forouhi et al } \\
\text { (2016) }\end{array}$ & $\begin{array}{l}\text { Effects of vitamin D2 or D3 } \\
\text { supplementation on glycaemic control } \\
\text { and cardiometabolic risk among people } \\
\text { at risk of type } 2 \text { diabetes: results of a } \\
\text { randomized double-blind placebo- } \\
\text { controlled trial }\end{array}$ & Inglaterra & $\begin{array}{l}\text { Diabetes, } \\
\text { Obesity and } \\
\text { Metabolism }\end{array}$ & $\begin{array}{l}\text { Ensaio Clinico } \\
\text { Randomizado }\end{array}$ & PubMed \\
\hline $\begin{array}{l}\text { Ryu et al } \\
\text { (2014) }\end{array}$ & $\begin{array}{l}\text { The effect of high-dose vitamin D } \\
\text { supplementation on insulin resistance } \\
\text { and arterial stiffness in patients with } \\
\text { type } 2 \text { diabetes }\end{array}$ & $\begin{array}{l}\text { Coreia do } \\
\text { Sul }\end{array}$ & $\begin{array}{l}\text { The Korean } \\
\text { Journal of } \\
\text { Internal } \\
\text { Medicine }\end{array}$ & $\begin{array}{l}\text { Ensaio Clinico } \\
\text { Randomizado }\end{array}$ & PubMed \\
\hline $\begin{array}{l}\text { Sollid et al } \\
\text { (2014) }\end{array}$ & $\begin{array}{l}\text { No Effect of High-Dose Vitamin D } \\
\text { Supplementation on Glycemic Status or } \\
\text { Cardiovascular Risk Factors in Subjects } \\
\text { With Prediabetes }\end{array}$ & Noruega & $\begin{array}{l}\text { American } \\
\text { Diabetes } \\
\text { Association }\end{array}$ & $\begin{array}{l}\text { Ensaio Clinico } \\
\text { Randomizado }\end{array}$ & PubMed \\
\hline $\begin{array}{l}\text { Nikooyeh et } \\
\text { al (2013) }\end{array}$ & $\begin{array}{l}\text { Daily intake of vitamin D- or calcium- } \\
\text { vitamin D-fortified Persian yogurt drink } \\
\text { (doogh) attenuates diabetes-induced } \\
\text { oxidative stress: evidence for } \\
\text { antioxidative properties of vitamin D }\end{array}$ & Irã & $\begin{array}{l}\text { Journal of } \\
\text { Human } \\
\text { Nutrition and } \\
\text { Dietetics }\end{array}$ & $\begin{array}{l}\text { Ensaio Clinico } \\
\text { Randomizado }\end{array}$ & PubMed \\
\hline $\begin{array}{c}\text { Heravifard et } \\
\text { al (2014) }\end{array}$ & $\begin{array}{l}\text { Regular Consumption of Both Vitamin } \\
\text { D- and Calciumand Vitamin D- } \\
\text { Fortified Yogurt Drink Is Equally } \\
\text { Accompanied by Lowered Blood } \\
\text { Lipoprotein (a) and Elevated Apoprotein } \\
\text { A1 in Subjects with Type } 2 \text { Diabetes: A } \\
\text { Randomized Clinical Trial }\end{array}$ & Irã & $\begin{array}{l}\text { Journal of the } \\
\text { American } \\
\text { College of } \\
\text { Nutrition }\end{array}$ & $\begin{array}{l}\text { Ensaio Clinico } \\
\text { Randomizado }\end{array}$ & PubMed \\
\hline $\begin{array}{c}\text { Shab-Bidar et } \\
\text { al (2011) }\end{array}$ & $\begin{array}{l}\text { Regular consumption of vitamin D- } \\
\text { fortified yogurt drink (Doogh) improved } \\
\text { endothelial biomarkers in subjects with } \\
\text { type } 2 \text { diabetes: a randomized double- } \\
\text { blind clinical trial }\end{array}$ & Irã & BMC Medicine & $\begin{array}{l}\text { Ensaio Clinico } \\
\text { Randomizado }\end{array}$ & PubMed \\
\hline
\end{tabular}




\begin{tabular}{|c|c|c|c|c|c|}
\hline $\begin{array}{l}\text { Zittermann et } \\
\text { al (2018) }\end{array}$ & $\begin{array}{l}\text { Daily Supplementation with } 4000 \text { IU } \\
\text { Vitamin D3 for Three Years Does Not } \\
\text { Modify Cardiovascular Risk Markers in } \\
\text { Patients with Advanced Heart Failure: } \\
\text { The Effect of Vitamin D on Mortality in } \\
\text { Heart Failure Trial }\end{array}$ & Alemanha & $\begin{array}{c}\text { Annals of } \\
\text { nutrition \& } \\
\text { metabolism }\end{array}$ & $\begin{array}{l}\text { Ensaio Clinico } \\
\text { Randomizado }\end{array}$ & EBSCOhost \\
\hline $\begin{array}{c}\text { Ebrahimkhani } \\
\text { et al (2010) }\end{array}$ & $\begin{array}{l}\text { The effects of vitamin D and } \\
\text { curcuminoids supplementation on } \\
\text { anthropometric measurements and blood } \\
\text { pressure in type } 2 \text { diabetic patients with } \\
\text { coexisting hypovitaminosis D: A } \\
\text { double-blind, placebocontrolled } \\
\text { randomized clinical trial }\end{array}$ & Irã & $\begin{array}{c}\text { Clinical } \\
\text { Nutrition } \\
\text { ESPEN }\end{array}$ & $\begin{array}{l}\text { Ensaio Clinico } \\
\text { Randomizado }\end{array}$ & Science Direct \\
\hline $\begin{array}{l}\text { Omidian et al } \\
\qquad(2019)\end{array}$ & $\begin{array}{l}\text { Effects of vitamin D supplementation on } \\
\text { circulatory YKL- } 40 \text { and MCP1 } \\
\text { biomarkers associated with vascular } \\
\text { diabetic complications: A randomized, } \\
\text { placebo-controlled, } \\
\text { clinical trial }\end{array}$ & Irã & $\begin{array}{l}\text { Diabetes \& } \\
\text { Metabolic } \\
\text { Syndrome: } \\
\text { Clinical } \\
\text { Research \& } \\
\text { Reviews }\end{array}$ & $\begin{array}{c}\text { Ensaio Clinico } \\
\text { Randomizado }\end{array}$ & Science Direct \\
\hline $\begin{array}{c}\text { Kalishdi et al } \\
\text { (2014) }\end{array}$ & $\begin{array}{l}\text { Effects of vitamin D supplementation on } \\
\text { insulin resistance and cardiometabolic } \\
\text { risk factors in children with metabolic } \\
\text { syndrome: a triple-masked controlled } \\
\text { trial }\end{array}$ & Irã & $\begin{array}{l}\text { Jornal de } \\
\text { pediatria }\end{array}$ & $\begin{array}{c}\text { Ensaio Clinico } \\
\text { Randomizado }\end{array}$ & Science Direct \\
\hline
\end{tabular}

Fonte: Autores (2021).

Depois dessa sistematização, foram analisados os artigos mediante a efetividade na eficácia ao melhorar do risco cardiovascular após uso de vitamina D. Dos 13 estudos selecionados, todos apresentam algum tipo de resultado relevante para a finalidade da patologia abordada.

Em seguida, foi realizada uma leitura integral dos estudos selecionados juntamente com a sistematização de acordo com a evolução dos achados clínicos do paciente, sendo classificados em: aumento dos níveis de vitamina $\mathrm{D}$, diminuição do colesterol total, diminuição dos níveis glicêmicos, diminuição dos níveis pressóricos e diminuição de formação de placas arterioscleróticas, conforme Quadro 2.

Quadro 2 - Principais benefícios do uso da vitamina D na diminuição do risco cardiovascular.

\begin{tabular}{|l|c|c|}
\hline \multicolumn{1}{|c|}{ PRINCIPAIS BENEFÍCIOS IDENTIFICADOS } & N & (\%) \\
\hline Aumento dos níveis de vitamina D & 13 & $100 \%$ \\
\hline Melhor controle glicêmico & 3 & $38 \%$ \\
\hline Diminuição nos níveis de PTH formação de células espumosas & 1 & $23 \%$ \\
\hline $\begin{array}{l}\text { Diminuição de } \\
\text { arterioscleróticas }\end{array}$ & 1 & $7 \%$ \\
\hline Diminuição dos níveis dos triglicerídeos & 2 & $7 \%$ \\
\hline Diminuição na rigidez arterial & & $15 \%$ \\
\hline
\end{tabular}

Fonte: Autores (2021). 
Apesar dos achados, todos os artigos fomentam a necessidade de realizar mais estudos para melhor elucidação das doses a serem utilizadas e mecanismos de ação com relação à utilização de vitamina D na diminuição do risco cardiovascular em pacientes diabéticos.

Após isso, procedeu-se com a classificação do nível de evidência dos ensaios clínicos randomizados que fazem parte da amostra final do estudo. Todos os estudos possuem elevado nível de evidência.

Quadro 3 - Classificação do nível de evidência dos estudos selecionados.

\begin{tabular}{|c|c|c|c|c|c|}
\hline Autor/Ano & $\begin{array}{c}\text { Limitação } \\
\text { metodológica }\end{array}$ & $\begin{array}{l}\text { Inconsistência } \\
\text { nos resultados }\end{array}$ & $\begin{array}{l}\text { Evidência } \\
\text { indireta }\end{array}$ & $\begin{array}{l}\text { Imprecisão dos } \\
\text { achados }\end{array}$ & $\begin{array}{l}\text { Nível de } \\
\text { evidência }\end{array}$ \\
\hline $\begin{array}{l}\text { Angelloti et al } \\
\text { (2018) }\end{array}$ & Não identificado & Não identificado & Não identificado & Não identificado & Alto \\
\hline Zaher et al (2018) & Não identificado & Não identificado & Não identificado & Não identificado & Alto \\
\hline E. Riek et al (2017) & Não identificado & Não identificado & Não identificado & Não identificado & Alto \\
\hline Forouhi et al (2016) & Não identificado & Não identificado & Não identificado & Não identificado & Alto \\
\hline Ryu et al (2014) & Não identificado & Não identificado & Não identificado & Não identificado & Alto \\
\hline Sollid et al (2014) & Não identificado & Não identificado & Não identificado & Não identificado & Alto \\
\hline $\begin{array}{l}\text { Nikooyeh et al } \\
\text { (2013) }\end{array}$ & Não identificado & Não identificado & Não identificado & Não identificado & Alto \\
\hline $\begin{array}{l}\text { Heravifard et al } \\
\qquad(2014)\end{array}$ & Não identificado & Não identificado & Não identificado & Não identificado & Alto \\
\hline $\begin{array}{c}\text { Shab-Bidar et al } \\
\text { (2011) }\end{array}$ & Não identificado & Não identificado & Não identificado & Não identificado & Alto \\
\hline $\begin{array}{l}\text { Zittermann et al } \\
\qquad \text { (2018) }\end{array}$ & Não identificado & Não identificado & Não identificado & Não identificado & Alto \\
\hline $\begin{array}{c}\text { Ebrahimkhani et al } \\
(2010)\end{array}$ & Não identificado & Não identificado & Não identificado & Não identificado & Alto \\
\hline $\begin{array}{l}\text { Omidian et al } \\
\text { (2019) }\end{array}$ & Não identificado & Não identificado & Não identificado & Não identificado & Alto \\
\hline $\begin{array}{l}\text { Kalishdi et al } \\
\text { (2014) }\end{array}$ & Não identificado & Não identificado & Não identificado & Não identificado & Alto \\
\hline
\end{tabular}

Fonte: Autoria própria (2021).

\section{Discussão}

O presente estudo revelou efeitos favoráveis associados à suplementação dietética com vitamina D. De modo geral, a revisão sistemática dos estudos observacionais apontou a vitamina supracitada como um fator capaz de alterar dos marcadores 
de risco cardiovascular em pacientes portadores de diabetes mellitus tipo II, que por consequência acarreta em uma diminuição de complicações microvasculares (Shab-Bidar et al., 2011; Omidian et al., 2019).

Ensaio clínico realizado anteriormente nos Estados Unidos, entre 2013 e 2014, com 127 pacientes randomizados para o uso de vitamina D3 $(n=66)$ e placebo $(n=61)$, demostrou que na vigésima quarta semana de estudo, os pacientes do grupo controle apresentavam concentrações plasmáticas de $25(\mathrm{OH}) \mathrm{D}$ de $47,1 \mathrm{ng} / \mathrm{mL}$ contra 25,5 ng/mL do grupo placebo. Além disso, entre os pacientes que não usavam medicações para colesterol (n=32); ocorreu uma diminuição do triglicerídeos na semana 24 e semana 48. Mudanças favoráveis também estiveram presentes nos níveis de HDL, relação TG/HDL e risco cardiovascular. Contudo, em pacientes que estavam fazendo uso de tratamento medicamentoso para colesterol os achados não foram significativos. Com isso, foi sugerido que o uso da vitamina $\mathrm{D}$ possuis maior significado em pacientes que não necessitam fazer terapia medicamentosa para colesterol (Angellotti et al., 2019).

Um outro ensaio estadunidense foi executado com 18 pacientes, cujo uso de medicamentos anti-hipertensivos foi eliminando durante o estudo, sendo todos os pacientes possuidores de diabetes mellitus controlada. Após três semanas de intervenção, os níveis de 1,25 (OH)2D aumentou significativamente em pacientes que fizeram uso da suplementação de calcitriol, porém nada ocorreu naqueles que fizeram uso de placebo. Não houve diminuição do mecanismo renina-angiotensinaaldosterona, entretanto ocorreu um aumento na expressão de receptores de vitamina D (Zaheer et al., 2018).

Adicionalmente, em um ensaio também estadunidense foram selecionados pacientes de vários pacientes a partir de 26 estudos longitudinais. Com isso, foi realizada uma suplementação de vitamina D que ao longo dos 4 meses resultou no aumento dos níveis de vitamina D no grupo controle, com $73 \%$ dos pacientes atingindo um nível superior a $\geq 30 \mathrm{ng} / \mathrm{mL}$ no estágio final. Além do mais, a suplementação de vitamina D3 diminuiu significativamente a absorção de colesterol em 50\% dos pacientes em um comparativo com o grupo placebo. Também foi observado que em macrofágos com insuficiência de vitamina D era existente uma porcentagem de colesterol 8 vezes maior, sugerindo que a suficiência de vitamina D diminui a captação de colesterol, esterificação de colesterol e formação de células espumosas mostrando bons resultados na melhora da rigidez arterial (Riek et al., 2018).

Já um ensaio clínico inglês analisou 340 pacientes, sendo 114 do grupo placebo, 112 do grupo vitamina D2 e 114 do grupo vitamina D3. As porcentagens de indíviduos com concentração de $25(\mathrm{OH}) \mathrm{D}<50 \mathrm{nmol} / \mathrm{ml}$ no início do estudo era de $58,8 \%, 50,9 \%$ e 50,9\% no grupo placebo, D2 e D3, respectivamente; na medida seguinte esses perdentuais foram de 47,3\%, 4,5\% e 3,5\%. Outra análise feita foi a diferença do controle glicêmico atraves da HbAlc, que não mostrou desfecho significativo entre os grupos placebo e suplementação com D2 e D3. Entre os resultados secundários, também não houve diferenças significativas em resultados secundários como: medidas antropométricas; pressão arterial e marcadores hepáticos. Entretanto, no tocante a diminuição na rigidez arterial ocorreram achados significativos no estudo (Forouhi et al., 2016);

Em um trabalho Sul Coreano foram randomizados 81 pacientes, em que 41 participaram do grupo placebo e 40 do grupo controle. No final do estudo o nível de vitamina D no grupo que sofreu a intervenção estava maior em relação ao grupo placebo. Não ocorreu mudanças nos níveis de HbA1c, perfil lipídico, PTH, assim como também não houve melhora na rigidez arterial entre os grupos (Ryu et al., 2014).

Na Noruega foi realizada um estudo contando com 484 participantes, sendo metade do grupo placebo e metade do grupo controle. Com o seguimento do estudo e aumento das concentrações séricas de $25(\mathrm{OH}) \mathrm{D}$ no grupo controle foi constatada uma diminuição do IMC, glicemia de jejum e glicemia após 2 horas da refeição e HbAlc em pacientes diabéticos, porém para pacientes pré-diabéticos esse resultado não possui a mesma expressão. Além disso, nos grupos que receberam vitamina D ocorreu diminuição nos níveis de PTH fazendo contraponto ao grupo placebo que possuiu um aumento nos seus níveis, porém não foi visto o mesmo resultado expressivo para os níveis pressóricos ao comparar os dois grupos. No quesito triglicerídeos e níveis de LDL ocorreu uma discreta diminuição desses indicadores em pacientes que não faziam tratamento medicamentoso e participaram 
do grupo controle, entretanto, para pacientes que já estavam sobre tratamento medicamentoso não foram observados resultados expressivos (Sollid et al., 2014).

Estudos realizados no Irã, que trabalharam com ingestão alimentar de iorgute persa fortificado com vitamina D e pacientes com diabetes mellitus demostraram que após a utilização do alimento, o grupo que fez a suplementação apresentou um aumento nos níveis séricos de vitamina D algo não encontrado no grupo placebo. O principal achado no estudo de Nikooyeh et al. (2013) foi a diminuição do estresse oxidativo ocorrido nos vasos pela diabetes, achado esse encontrado em suma maioria no grupo que fez uso do iorgute fortificado com vitamina D. Já o estudo de Heravifard et al. (2013) concluiu que não houve mudança significativa em marcadores como colesterol sérico, nos níveis de LDL e HDL dentro dos grupos insulino dependentes; porém, no quesito resistência a insulina e diminuição da lipoproteina Apo A1 foi notado uma diferença significativa entre os pacientes que realizaram a suplementação alimentar e os pacientes que utilizaram iorgute não fortificado, mostrando melhores valores no primeiro grupo (Heravifard et al., 2013; Nikooyeh et al., 2013)

Um outro estudo iraniano que também trabalhou com a ingesta de iorgute fortificado com vitamina D (Doogh) foi realizado com 75 pacientes, sendo divididos entre aqueles que iriam consumir apenas o iorgute não fortificado (35) e os que utilizariam o alimento suplementado $(\mathrm{n}=40)$. De imediato, o estudo demonstrou aumento nos níveis de vitamina $\mathrm{D}$ em 37 dos pacientes que aderiram a suplementação, contra apenas 2 dos pacientes que utilizaram alimentação comum, junto a esse evento, também foi observada a diminuição dos níveis séricos de PTH (Shab-Bidar et al., 2011).

Ao avaliar as medidas antropométricas (IMC e circunferência abdominal) não foi observada diferença entre os grupos, entretanto, ao serem analisados os níveis pressóricos sistólicos e diastólicos, foi encontrado uma pequena porém significativa diminuição em comparação aos valores iniciais no grupo que realizou a ingesta alimentar. No quesito estado glicêmico, foi constatada uma diferença positiva entre os grupos, em que os pacientes que realizaram a adesão possuíram melhores valores. Ao avaliar os marcadores endoteliais (E-selectina e MMP-9) foi constatada uma diminuição significativa no grupo controle (ShabBidar et al., 2011).

Um estudo foi produzido na Alemaha em que todos os pacientes eram afetados por cardiomiopatia dilatada ou isquêmica, com grande maioria dos pacientes com fração de ejeção menor que 30\%. Esses pacientes foram divididos em grupo placebo e o grupo que ira receber a suplementação diária de 4000 UI de vitamina D3 por por três anos. A princípio foi observado um aumento nos níveis de HDL no grupo que sofreu a intervenção, dado esse não observado no tocante ao não-HDL e LDL de forma isolada, ficando os dois grupos em pé de igualdade. Também não foram encontradas mudanças nos níveis de triglicerídeos nos pacientes dos dois grupos, assim não havendo mudança nos parâmetros lipídicos que pudessem afetar o risco cardiovascular no perfil dos pacientes presentes no estudo (Zittermann et al., 2018).

Em ensaio clínico produzido no Irã com 80 pacientes com DM2, sendo divididos em grupos placebo, vitamina D, curcuminóides (potente anti-inflamatório) e associação entre curcuminoide e vitamina D. Foi constando melhores níveis séricos de vitamina D em pacientes que realizaram suplementação exclusiva de vitamina D. Sobre a pressão arterial sistólica e a pressão arterial diastólica foi presenta melhora nos valores em pacientes que estavam realizando o tratamento tanto com vitamina $\mathrm{D}$, tanto com curcuminóides e o composto misto (vitamina D e curminóides), entretanto, ocorreu melhora mais significativa no grupo vitamina D. Contudo, não foram encontrados achados significativos no tocante a diminuição do IMC e da circunferência abdominal (Ebrahimkhani; Ghavamzadeh \& Mehdizadeh, 2020).

Em um estudo realizado com 48 pacientes, foram divididos em dois grupos de igual número de participantes, em que se utilizou vitamina $\mathrm{D}$ em um grupo e placebo no outro. No início do estudo, não foram notadas diferenças significativas entre características gerais (valores antropométricos, pressão arterial e índices metabólicos), nem tão pouco nos níveis de insulina de jejum. No entanto, ocorreu uma ligeira diferença entre os valores de HbAlc, sendo o grupo que fez suplementação obtendo valores mais expressivos. Além disso, também houve diminuição significativa nos biomarcadores inflamatórios e nos fatores 
metabólicos IL-6 e Homar-IR (medida que aparece no resultado do exame de sangue que serve para avaliar a resistência à insulina) no grupo que sofreu intervenção após 3 meses (Omidian et al., 2019).

Em um trabalho publicado na Sociedade Brasileira de Pediatria foi analisado através de ensaio clinico o uso da vitamina D sobre a resistência à insulina e o risco cardiometabólico em crianças com diabetes mellitus 1 . Foi realizado o estudo sobre dois grupos, um com 21 participantes que sofreram intervenção e o outro com 22 que utilizaram placebo. Após o ensaio, no grupo vitamina $\mathrm{D}$ foi percebida uma redução nos níveis de triglicerídeos em comparação a linha de base adotada pelo estudo e também em comparação ao grupo placebo. Uma diminuição foi observada também nos níveis de insulina sérica e no marcador de resistência à insulina HOMAR-IR em pacientes que fizeram uso do suplemento. Entretanto, não foi observado diminuição nos níveis de colesterol total, LDL-C e HDL-C em ambos os grupos (Kelishadi et al., 2014).

\section{Considerações Finais}

A maioria dos estudos selecionados demostrou alguma eficiência nos marcadores adotados nos ensaios. Dentro os achados destacaram-se: aumento dos níveis de vitamina D, melhor controle glicêmico, diminuição nos níveis de PTH, diminuição de formação de células espumosas arterioscleróticas, diminuição dos níveis dos triglicerídeos, e diminuição na rigidez arterial; o que reforça a ideia de que o uso desta vitamina representa uma alternativa promissora na redução de complicações cardiovasculares em portadores de diabetes mellitus tipo II, exercendo um impacto positivo no âmbito da saúde pública.

Entretanto, ressalta-se que, por mais que a função benéfica dessa vitamina já seja conhecida pela comunidade científica, ainda existem muitas lacunas a serem preenchidas acerca de sua utilização. Há necessidade de realizar mais ensaios para definir qual a posologia ideal da substância, os reais benefícios que ela pode trazer em longo prazo, os possíveis efeitos colaterais e os cuidados necessários para evitar uma possível toxicidade, na tentativa de trazer evidências mais robustas que sustentem sua utilização. É fundamental que essas questões sejam esclarecidas devido ao baixo custo e facilidade de acesso à vitamina $\mathrm{D}$, para que assim o tratamento possa ser otimizado e a morbimortalidade seja efetivamente reduzida.

Por fim, devem ser realizados mais trabalhos que tenha por finalidade o estudo da diminuição de eventos cardiovasculares catastróficos correlacionado com o uso de vitamina D, para assim, ter uma correlação direta entre a suplementação vitamínica em estudo e o desfecho cardiovascular.

\section{Referências}

Annweiler, C., Dursun, E., Féron, F., Gezen-Ak, D., Kalueff, A. V., Littlejohns, T., Llewellyn, D. J.; Millet, P., Scott, T., \& Tucker, K. L. (2014) Vitamin D and cognition in older adults': updated international recommendations. Journal Of Internal Medicine, 277(1), 45-57. http://dx.doi.org/10.1111/joim.12279.

Angellotti, E., D'alessio, D., Dawson-Hughes, B., Chu, Y., Nelson, J., Hu, P., Cohen, R. M., \& Pittas, A. G. (2019) Effect of vitamin D supplementation on cardiovascular risk in type 2 diabetes. Clinical Nutrition, 38(5), 2449-2453. http://dx.doi.org/10.1016/j.clnu.2018.10.003.

Aguiar, C., Duarte, R., Carvalho, D. (2019) Nova abordagem para o tratamento da diabetes: da glicemia à doença cardiovascular. Revista Portuguesa de Cardiologia, 38(1), 53-63. https://doi.org/10.1016/j.repc.2018.03.013

Carrillo-Larco, R. M. \& Bernabé-Ortiz, A. (2019) Diabetes Mellitus Tipo 2 En Perú: Una Revisión Sistemática Sobre La Prevalencia E Incidencia En Población General. Rev Peru Med Exp Salud Publica, 36(1), 26-36. http://dx.doi:10.17843/rpmesp.2019.361.4027.

Ebrahimkhani, S., Ghavamzadeh, \& S., Mehdizadeh, A. (2020) The effects of vitamin D and curcuminoids supplementation on anthropometric measurements and blood pressure in type 2 diabetic patients with coexisting hypovitaminosis D: a double-blind, placebo-controlled randomized clinical trial. Clinical Nutrition Espen, 37, 178-186. http://dx.doi.org/10.1016/j.clnesp.2020.02.017.

Forouhi, N. G., Menon, R. K., Sharp, S. J.; Mannan, N., Timms, P. M.; Martineau, A. R., Rickard, A. P., Boucher, B. J., Chowdhury, T. A., \& Griffiths, C. J. (2016) Effects of vitamin D2 or D3 supplementation on glycaemic control and cardiometabolic risk among people at risk of type 2 diabetes: results of a randomized double-blind placebo-controlled trial. Diabetes, Obesity And Metabolism, 18(4), 392-400. http://dx.doi.org/10.1111/dom.12625.

Galvão, M. C. B.; \& Ricarte, I. L. M. (2019) Revisão Sistemática Da Literatura: conceituação, produção e publicação. Logeion: Filosofia da Informação, 6(1), 57-73. http://dx.doi.org/10.21728/logeion.2019v6n1.p57-73.

Heravifard, S.; Neyestani, T. R.; Nikooyeh, B.; Alavi-Majd, H.; Houshiarrad, A.; Kalayi, A.; Shariatzadeh, N.; Zahedirad, M.; Tayebinejad, N.; \& Salekzamani, S. (2013) Regular Consumption of Both Vitamin D- and Calcium- and Vitamin D-Fortified Yogurt Drink Is Equally Accompanied by Lowered Blood 
Lipoprotein (a) and Elevated Apoprotein A1 in Subjects with Type 2 Diabetes: a randomized clinical trial. Journal Of The American College Of Nutrition, 32(1), 26-30. http://dx.doi.org/10.1080/07315724.2013.767659.

Hirschler, V., Maccallini, G., Sanchez, M., Claudio, G., Molinari, C., Figueroa, M., Arnada, C., \& Hidalgo, M. (2015) Improvement of Apolipoprotein B in Argentine Indigenous School Children after Vitamin D Supplementation. Cardiovascular \& Hematological Agents In Medicinal Chemistry. 13 (2), 137-145. http://dx.doi.org/10.2174/1871525712666140516102319.

Liefaard, M. C., Ligthart, S., Vitezova, A., Hofman, A., Uitterlinden, A. G., Jong, J. C. K., Franco, O. H., Zillikens, M. C., \& Dehghan, A. (2015) Vitamin D and C-Reactive Protein: a mendelian randomization study. Plos One, 10(7), 1-12. http://dx.doi.org/10.1371/journal.pone.0131740.

Lu, Y., Hajifathalian, K., Ezzati, M., Woodward, M., Rimm, E. B., \& Danaei, G. (2014) Metabolic mediators of the effects of body-mass index, overweight, and obesity on coronary heart disease and stroke: a pooled analysis of 97 prospective cohorts with 1.8 million participants. The Lancet, 383 (9921), 970-983. http://dx.doi.org/10.1016/s0140-6736(13)61836-x.

Kelishadi, R., Salek, S., Salek, M., Hashemipour, M., \& Movahedian, M. (2014) Effects of vitamin D supplementation on insulin resistance and cardiometabolic risk factors in children with metabolic syndrome: a triple-masked controlled trial. Jornal de Pediatria (Versão em Português), 90(1), 28-34. http://dx.doi.org/10.1016/j.jpedp.2013.06.005.

Muscogiuri, G., Altieri, B., Annweiler, C., Balercia, G., Pal, H. B., Boucher, B. J., Cannell, J. J., Foresta, C., Grübler, M. R., \& Kotsa, K. (2016) Vitamin D and chronic diseases: the current state of the art. Archives Of Toxicology, 91(1). 97-107. http://dx.doi.org/10.1007/s00204-016-1804-x.

Ministério da Saúde. Secretaria de Ciência, Tecnologia e Insumos Estratégicos. Departamento de Ciência e Tecnologia. Diretrizes metodológicas: Sistema GRADE - Manual de graduação da qualidade da evidência e força de recomendação para tomada de decisão em saúde: Ministério da Saúde; 2014. http://bvsms.saude.gov.br/bvs/publicacoes/diretrizes_metodologicas_sistema_grade.pdf

Mcdonnell, S. L., Baggerly, C., French, C. B., Baggerly, L. L., Garland, C. F., Gorham, E. D., Lappe, J. M., \& Heaney, R. P. (2016) Serum 25-Hydroxyvitamin D Concentrations $\geq 40 \mathrm{ng} / \mathrm{ml}$ Are Associated with $>65 \%$ Lower Cancer Risk: pooled analysis of randomized trial and prospective cohort study. Plos One, 11(4), 1-15. http://dx.doi.org/10.1371/journal.pone.0152441.

Nikooyeh, B., Neyestani, T. R., Tayebinejad, N., Alavi-Majd, H., Shariatzadeh, N., Kalayi, A., Zahedirad, M., Heravifard, S., \& Salekzamani. (2013) S. Daily intake of vitamin D- or calcium-vitamin D-fortified Persian yogurt drink (doogh) attenuates diabetes-induced oxidative stress: evidence for antioxidative properties of vitamin d. Journal Of Human Nutrition And Dietetics, 27, 276-283, 5. http://dx.doi.org/10.1111/jhn.12142.

Nakashima, A., Yokoyama, K., Yokoo, T., \& Urashima, M. (2016) Role of vitamin D in diabetes mellitus and chronic kidney disease. World Journal Of Diabetes, 7(5), 89-100, http://dx.doi.org/10.4239/wjd.v7.i5.89.

Omidian, M., Mahmoudi, M., Javanbakht, M. H., Eshraghian, M. R., Abshirini, M., Daneshzad, E., Hasani, H., Alvandi, E., \& Djalali, M. (2019) Effects of vitamin D supplementation on circulatory YKL-40 and MCP-1 biomarkers associated with vascular diabetic complications: a randomized, placebo-controlled, double-blind clinical trial. Diabetes \& Metabolic Syndrome: Clinical Research \& Reviews, 13(5), 2873-2877. http://dx.doi.org/10.1016/j.dsx.2019.07.047.

Odutayo, A., Wong, Christopher X; Hsiao, Allan J., Hopewell, Sally; A., Douglas, G.; \& A Emdin, C. (2016) Atrial fibrillation and risks of cardiovascular disease, renal disease, and death: systematic review and meta-analysis. BMJ, 6(5), 1-11. http://dx.doi.org/10.1136/bmj.i4482.

PRISMA Group. Preferred Reporting Items for Systematic Reviews and Meta-Analyses. (s.1.): University of Ottawa/Oxford University, 2015. http://www.prisma-statement.org/PRISMAStatement/. Acesso em: 2 abr. 2018.

Ryu, O., Chung, W., Lee, S., Hong, K., Choi, M., \& Yoo, H. The effect of high-dose vitamin D supplementation on insulin resistance and arterial stiffness in patients with type 2 diabetes. (2014) The Korean Journal Of Internal Medicine, 29(5), 620-629. http://dx.doi.org/10.3904/kjim.2014.29.5.620.

Ruff, C. T., Giugliano, R. P., Braunwald, E., Hoffman, E. B., Deenadayalu, N., Ezekowitz, M. D., Camm, A. J., Weitz, J. I., Lewis, B. S., \& Parkhomenko, A. (2014) Comparison of the efficacy and safety of new oral anticoagulants with warfarin in patients with atrial fibrillation: a meta-analysis of randomised trials. The Lancet, 383(9921), 955-962. http://dx.doi.org/10.1016/s0140-6736(13)62343-0.

Riek, A. E., Oh, J., Darwech, I., Worthy, V., Lin, X., Ostlund, R. E., Zhang, R. M., \& Bernal-Mizrachi, C. (2018) Vitamin D3 supplementation decreases a unique circulating monocyte cholesterol pool in patients with type 2 diabetes. The Journal Of Steroid Biochemistry And Molecular Biology, 177, 187-192. http://dx.doi.org/10.1016/j.jsbmb.2017.09.011.

Sociedade Brasileira De Diabetes (SBD). (2019-2020) Diretrizes da Sociedade Brasileira de Diabetes. São Paulo: AC Farmacêutica, 2019. https://www.diabetes.org.br/profissionais/images/DIRETRIZES-COMPLETA-2019-2020.pdf

Strain, W. D. \& Paldánius, P. M. (2018) Diabetes, cardiovascular disease and the microcirculation. Cardiovasc Diabetol, $17(1), 57$. http://dx.doi.org/10.1186/s12933-018-0703-2

Shab-Bidar, S., Neyestani, T. R., Djazayery, A., Eshraghian, M., Houshiarrad, A., Gharavi, A., Kalayi, A., Shariatzadeh, N., Zahedirad, M., \& Khalaji, N. (2011) Regular consumption of vitamin D-fortified yogurt drink (Doogh) improved endothelial biomarkers in subjects with type 2 diabetes: a randomized double-blind clinical trial. Bmc Medicine, 9(1), 1-10. http://dx.doi.org/10.1186/1741-7015-9-125.

Steele, J. M., Urbina, E. M., Mazur, W. M., Khoury, P. R., Nagueh, S. F., Tretter, J. T., \& Alsaied, T. (2020) Left atrial strain and diastolic function abnormalities in obese and type 2 diabetic adolescents and young adults. Cardiovascular Diabetology, 19(1), 1043-1053. http://dx.doi.org/10.1186/s12933-020-01139-9.

Sharma, A., Zhao, X., Hammill, B. G., Hernandez, A. F., Fonarow, G. C., Felker, G. M., Yancy, C. W., Heidenreich, P. A., Ezekowitz, J. A., \& Devore, A (2018) D. Trends in Noncardiovascular Comorbidities Among Patients Hospitalized for Heart Failure. Circulation: Heart Failure, 11(6), 37-40. http://dx.doi.org/10.1161/circheartfailure.117.004646. 
Research, Society and Development, v. 10, n. 7, e49810716862, 2021

(CC BY 4.0) | ISSN 2525-3409 | DOI: http://dx.doi.org/10.33448/rsd-v10i7.16862

Verdoia, M., Nardin, M., Rolla, R., Negro, F., Gioscia, R., Afifeh, A. M. S., Viglione, F., Suryapranata, H., Marcolongo, M., \& Luca, G. (2020) Association of lower vitamin D levels with inflammation and leucocytes parameters in patients with and without diabetes mellitus undergoing coronary angiography. European Journal Of Clinical Investigation, 51(4), 134-139. http://dx.doi.org/10.1111/eci.13439.

Wacker, M., \& Holick, M. Vitamin D. (2013) Effects on Skeletal and Extraskeletal Health and the Need for Supplementation. Nutrients, 5, 111-148, 10 jan. http://dx.doi.org/10.3390/nu5010111.

Zittermann, A. (2018). Vitamin D Status, Supplementation and Cardiovascular Disease. Anticancer Research, 38(2), 1179-1186. http://dx.doi.org/10.21873/anticanres.12338.

Zaheer, S., Taquechel, K., Brown, J. M., Adler, G. K., Williams, J. S., \& Vaidya, A. (2018) A randomized intervention study to evaluate the effect of calcitriol therapy on the renin-angiotensin system in diabetes. Journal Of The Renin-Angiotensin-Aldosterone System, 19(1), 1-8. http://dx.doi.org/10.1177/1470320317754178.

Zittermann, A., Ernst, J. B., Prokop, S., Fuchs, U., Dreier, J., Kuhn, J., Knabbe, C., Börgermann, J.; Berthold, H. K., \& Pilz, S. (2018) Daily Supplementation with 4000 IU Vitamin D3 for Three Years Does Not Modify Cardiovascular Risk Markers in Patients with Advanced Heart Failure: the effect of vitamin d on mortality in heart failure trial. Annals Of Nutrition And Metabolism, 74(1), 62-68. http://dx.doi.org/10.1159/000495662. 